\title{
Kelly McKennon
}

\section{A family of Kučera spaces}

Czechoslovak Mathematical Journal, Vol. 29 (1979), No. 4, 503-513

Persistent URL: http://dml.cz/dmlcz/101632

\section{Terms of use:}

(C) Institute of Mathematics AS CR, 1979

Institute of Mathematics of the Czech Academy of Sciences provides access to digitized documents strictly for personal use. Each copy of any part of this document must contain these Terms of use.

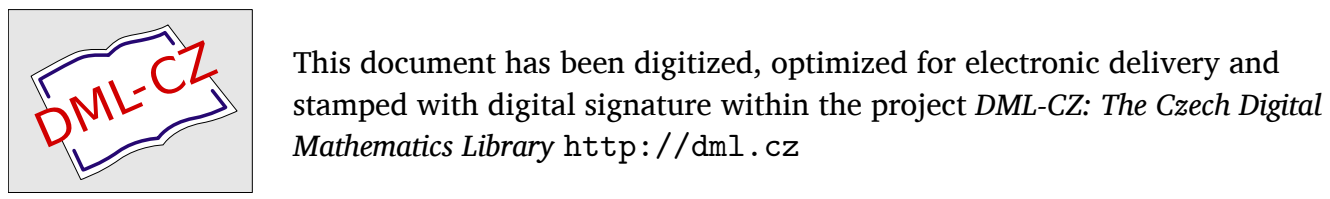




\title{
CZECHOSLOVAK MATHEMATICAL JOURNAL \\ Mathematical Institute of the Czechoslovak Academy of Sciences \\ V. 29 (104), PRAHA 19.12. 1979, No. 4
}

\section{A FAMILY OF KUCERA SPACES}

\author{
Kelly McKennon, Pullman
}

(Received February 25, 1972, in revised form May 31, 1979)

Let $N$ and $Z^{+}$be the sets of all positive and non-negative integers, respectively. Let $n$ be a fixed, but arbitrary, element of $N$. Write $\mathfrak{M}$ for the set of all functions $s \mid\{1,2, \ldots, n\} \rightarrow Z^{+}$and, for each $s \in \mathfrak{M}$, let

$$
|s|=\sum_{j=1}^{n} s_{j} .
$$

For each $m \in N$, let $\mathfrak{P}(m)$ be the set $\{(s, w) \in \mathfrak{M} \times \mathfrak{M}:|s|+|w| \leqq m\}$. For each $s \in \mathfrak{M}$, let $\theta_{s} \mid R^{n} \rightarrow R$ be defined by

$$
\theta_{s}(x)=(2 \pi i)^{|s|} \cdot \prod_{j=1}^{n} x_{j}^{s_{j}}
$$

for all $x \in R^{n}$. By $\lambda$ will be meant that version of Lebesgue measure on $R^{n}$ such that

$$
\int_{R^{n}} f \mathrm{~d} \lambda=\left(\frac{1}{\sqrt{ }(2 \pi)}\right)^{n} \cdot \int_{R^{n}} f(x) \mathrm{d} x
$$

for all integrable functions $f$ on $R^{n}$. For each $p \in[1, \infty],\|\|_{p}$ will be the usual $L_{p}$-norm on $L_{p}\left(R^{n}, \lambda\right)$. Other notation will be as in $[10]$.

The space $\mathfrak{S}$ of rapidly decreasing functions in $C^{\infty}\left(R^{n}\right)$ bears a topology induced by the family $\left\{\|\|_{(s, w)}: s, w \in \mathfrak{M}\right\}$ of semi-norms where

$$
\|f\|_{(s, w)}=\left\|\theta_{s} \cdot D^{w}(f)\right\|_{\infty}
$$

for all $f \in \mathfrak{S}$ and $s, w \in \mathfrak{M}$. Kucera in [3] introduced a novel construction of $\mathfrak{S}$. He took a sequence $\left\{H_{m}\right\}_{m=0}^{\infty}$ of locally convex spaces with the properties:

(1) $H_{m-1} \supset H_{m}$ and the identity map on $H_{m}$ is continuous for all $n \in N$;

(2) $\subseteq$ is a dense subset of each $H_{m}$;

(3) $\subseteq=\bigcap_{m=0}^{\infty} H_{m}$ and the topology on $\subseteq$ is the projective topology induced by the spaces $H_{m}$. 
For each $m$ and $k$ in $Z^{+}$, he defined $\mathfrak{D}_{k, m}$ to be the set of multiplication operators from $H_{k}$ into $H_{m}$ and attached to it the usual operator norm. The spaces $\mathfrak{D}_{k, m}$ were shown to be Banach spaces, $\mathfrak{D}_{k, m}$ was proved to be just $\{0\}$ if $k<m$, and the linear space $\bigcap_{m=0}^{\infty} \bigcup_{k=0}^{\infty} \mathfrak{D}_{k, m}$ was exhibited to be precisely $\mathfrak{D}_{M}$, the space of slowly increasing functions in $C^{\infty}\left(R^{n}\right)$. In [5], the usual topology on $\mathfrak{D}_{M}$ was shown to agree with the projective topology on $\bigcap_{m=0}^{\infty} \bigcup_{k=0}^{\infty} \mathfrak{D}_{k, m}\left(\bigcup_{k=0}^{\infty} \mathfrak{D}_{k, m}\right.$ bearing its inductive topology for each $m$ ). In [6], these results were exploited to show that the natural mapping from $\mathfrak{D}_{M} \times \mathfrak{S}^{\prime}$ onto $\mathfrak{S}^{\prime}$ is jointly continuous.

A concrete sequence $\left\{H_{m}\right\}_{n=0}^{\infty}$ was studied by KuCERA in [3] and [4]. Here $H_{0} \equiv$ $\equiv L_{2}\left(R^{n}\right)$ and, for each $m \in N, H_{m} \equiv\left\{f \mid R^{n} \rightarrow C: \theta_{s} . D^{w}(f) \in H_{0}\right.$ for all $(s, w) \in$ $\in \mathfrak{P}(m)\}$. The topology on $H_{m}$ was induced by the norm given by

$$
\|f\|_{m}=\left(\sum_{(s, w) \in \mathfrak{F}_{(m)}}\left\|\theta_{s} \cdot D^{w}(f)\right\|_{2}^{2}\right)^{1 / 2}
$$

and $H_{m}$ was seen to be a Hilbert space. The Fourier transform was shown to be a surjective linear isometry on $H_{m}$ and the spaces $\mathfrak{D}_{k, m}$ were studied in some detail.

Kucera's spaces suffer from some defects however. First of all, the functions in $H_{m}$ need not have classical derivatives of all orders less than $m$. Second, they may need not even be integrable, and so the definition of the Fourier transform is not so neat as might be wished. Third, the functions are not necessarily either bounded or continuous.

The present paper seeks to supply these deficiencies by introducing a related, but different, sequence $\left\{\mathfrak{H}_{m}\right\}_{m=0}^{\infty}$. The new spaces $\mathfrak{H}_{m}$ do not retain the property of being Hilbert spaces, but they are reflexive.

Let $\mathfrak{H}_{0}$ be the linear space $\left\{f \in L_{1}\left(R^{n}\right): \hat{f} \in L_{1}\left(R^{n}\right)\right\}$ where the Fourier Transform $\hat{f}$ is defined by

$$
\hat{f}(x)=\left(\frac{1}{\sqrt{ }(2 \pi)}\right)^{n} \cdot \int_{R^{n}} e^{-i\langle t, x\rangle} f(t) \mathrm{d} t
$$

for all $x \in R^{n}$ (here $\left.\langle t, x\rangle=\sum_{j=1} t_{j} x_{j}\right)$. For $f \in \mathfrak{H}_{0}$, let

$$
\|f\|^{(0)}=\|f\|_{1}+\|\hat{f}\|_{1} \text {. }
$$

Below are listed some properties of $\mathfrak{H}_{0}$ along with references:

(6) $\mathfrak{H}_{0}$ is a semi-simple Banach algebra under convolution ([7] 6.1.1);

(7) if $f^{*}(x)=f(-x)$ for all $x \in R^{n}$, then

(8) $\hat{\hat{f}}=f^{*}$ for all $f \in \mathfrak{H}_{0}([2] 31.17)$;

(9) the Fourier transform is a linear isometry of $\mathfrak{H}_{0}$ onto itself (obvious from (5), (7), and (8)); 
(10) $(f * h)^{\wedge}=\hat{f} . \hat{h}$ for all $f, h \in \mathfrak{H}_{0}([2] 31.5)$;

(11) $\mathfrak{H}_{0}$ is a dense ideal in $L_{1}\left(R^{n}\right)$ (under convolution) and $L_{1}\left(R^{n}\right) * \mathfrak{S}_{0}=\mathfrak{S}_{0}$ ([2] 39.34).

A function $f \mid R^{n} \rightarrow C$ is said to be positive-definite if $\sum_{j=1}^{m} \sum_{k=1}^{m} \alpha_{j} \bar{\alpha}_{k} f\left(x^{(j)}-x^{(k)}\right) \geqq 0$ whenever $\left\{\alpha_{j}\right\}_{j=1}^{m}$ are complex numbers and $\left\{x^{(j)}\right\}_{j=1}^{m}$ is a subset of $R^{n}$. Let $P\left(R^{n}\right)$ be the set of all continuous positive-definite functions on $R^{n}$ and $K\left(R^{n}\right)$ be the linear span of $P\left(R^{n}\right)$ in $C\left(R^{n}\right)$ (the elements of $K\left(R^{n}\right)$ are all bounded ([2] 32.4.11). Write $M\left(R^{n}\right)$ for the Banach algebra (under convolution) of all complex, bounded, regular Borel measures on $R^{n}$, the norm \|\|$_{M}$ on $M\left(R^{n}\right)$ being the total variation norm. Then

(12) the Fourier transform is an algebra isomorphism of $M\left(R^{n}\right)$ onto $K\left(R^{n}\right),\|\hat{\mu}\|_{\infty} \leqq$ $\leqq\|\mu\|_{M}$ for all $\mu \in M\left(R^{n}\right)$, and $P\left(R^{n}\right)=\left\{\hat{\mu}: \mu \geqq 0, \mu \in M\left(R^{n}\right)\right\}([2], 33.1$ and 33.3).

For each $f \in K\left(R^{n}\right)$, let $\mu_{f}$ be the measure in $M\left(R^{n}\right)$ such that $\hat{\mu}_{f}=f$.

Theorem 1. The set $\mathfrak{J}_{0}$ is an ideal in $K\left(R^{n}\right)$ and $K\left(R^{n}\right)$ is the family of multiplication operators on $\mathfrak{H}_{0}$. Furthermore, if $f \in K\left(R^{n}\right)$, then $\left\|\mu_{f}\right\|_{M}$ is the operator norm of $f$ viewed as a linear operator on $\mathfrak{H}_{0}$. Finally,

$$
\mathfrak{S}_{0}=K\left(R^{n}\right) \cap L_{1}\left(R^{n}\right)
$$

Proof. The first two assertions of Theorem 1 are just [7], Theorem 6.3.1, for $p=1$. The first of these assertions implies $\mathfrak{H}_{0} \subset K\left(R^{n}\right) \cap L_{1}\left(R^{n}\right)$. Let $f$ be any function in $K\left(R^{n}\right) \cap L_{1}\left(R^{n}\right)$. Then $\hat{\mu}_{f}=f$ is in $L_{1}\left(R^{n}\right)$ and so, by [2] 31.44.d, $\mu_{f}$ is absolutely continuous with respect to Lebesgue measure on $R^{n}$ and its Radon-Nikodým derivative $g$ has $f$ as its Fourier transform. By [2] 31.44.b, we have $\hat{f}=g^{*}$ which is in $L_{1}\left(R^{n}\right)$. Hence, $f$ is in $\mathfrak{H}_{0}$, which proves (13). Q.E.D.

Theorem 2. For all f, $g \in L_{\infty}\left(R^{n}\right)$, let $F_{f, g} \mid \mathfrak{H}_{0} \rightarrow C$ be defined by

$$
F_{f, g}(h)=\int_{R^{n}}(f h+g \hat{h}) \mathrm{d} \lambda
$$

for all $h \in \mathfrak{H}_{0}$. Then $\mathfrak{S}_{0}^{\prime}=\left\{F_{f, g}: f, g \in L_{\infty}\left(R^{n}\right)\right\}$.

Proof. It is well-known that $L_{\infty}\left(R^{n}\right)$ may be identified with $L_{1}\left(R^{n}\right)^{\prime}$. If $\mathfrak{H}_{0}$ is identified with the projective limit $\left\{(h, \hat{h}): h \in \mathfrak{H}_{0}\right\}$, then Theorem 2 follows from [9] IV.4.4. Q. E.D. 
A direct computation shows that $\|\hat{f}\|_{\infty} \leqq\|f\|_{1}$ for all $f \in L_{1}\left(R^{n}\right)$. Consequently, by $(8)$,

$$
\|f\|_{\infty} \leqq\|\hat{f}\|_{1} \leqq\|f\|^{(0)}
$$

Theorem 3. The Banach space $\mathfrak{H}_{0}$ is reflexive.

Proof. It will suffice to prove that the unit ball $B_{0}$ of $\mathfrak{H}_{0}$ is $\sigma\left(\mathfrak{H}_{0}, \mathfrak{S}_{0}^{\prime}\right)$ - compact. Let $\left\{f_{\alpha}\right\}$ be an arbitrary net in $B_{0}$. Alaoglu's Theorem ([2] B.25) implies that there exists a subnet $\left\{f_{r(\beta)}\right\}$ such that $\lim _{\beta} F\left(f_{r(\beta)}\right)$ exists for all $F \in \mathfrak{Y}_{0}^{\prime}$. For each $h \in L_{1}\left(R^{n}\right) \cap$ $\cap L_{\infty}\left(R^{n}\right)$, Theorem 2 implies that

$$
\lim _{\beta} \int_{R^{n}} h \cdot f_{r(\beta)} \mathrm{d} \lambda=\lim _{\beta} F_{h, 0}\left(f_{r(\beta)}\right)
$$

exists. Since $L_{1}\left(R^{n}\right) \cap L_{\infty}\left(R^{n}\right)$ is dense in $L_{1}\left(R^{n}\right)$, and since (14) implies

$$
\varlimsup_{\beta}\left\|f_{r(\beta)}\right\|_{\infty} \leqq \varlimsup_{\beta}\left\|f_{r(\beta)}\right\|^{(0)} \leqq 1,
$$

it follows that

$$
\lim _{\beta} \int_{R^{n}} h \cdot f_{r(\beta)} \mathrm{d} \lambda \text { exists for all } h \in L_{1}\left(R^{n}\right)
$$

Noting that

$$
\varlimsup_{\beta}\left\|\mu_{f_{r(\beta)}}\right\|_{M}=\varlimsup_{\beta}\left\|\hat{f}_{r(\beta)}\right\|_{1} \leqq \varlimsup_{\beta}\left\|f_{r(\beta)}\right\|^{(0)} \leqq 1,
$$

we may apply Theorem 6.2.ii and 6.5.iii of [8] to obtain some $f \in K\left(R^{n}\right)$ such that

$$
\lim _{\beta} \int_{R^{n}} h \cdot f_{r(\beta)} \mathrm{d} \lambda=\int_{R^{n}} h \cdot f \mathrm{~d} \lambda \text { for all } h \in L_{1}\left(R^{n}\right) .
$$

For each $h \in L_{1}\left(R^{n}\right) \cap L_{\infty}\left(R^{n}\right)$, Hölder's Inequality implies

$$
\begin{gathered}
\left|\int_{R^{n}} h \cdot f \mathrm{~d} \lambda\right|=\lim _{\beta}\left|\int_{R^{n}} h \cdot f_{r(\beta)} \mathrm{d} \lambda\right| \leqq \\
\leqq\|h\|_{\infty} \cdot \varlimsup_{\beta}\left\|f_{r(\beta)}\right\|_{1} \leqq\|h\|_{\infty} \cdot \varlimsup_{\beta}\left\|f_{r(\beta)}\right\|(0) \leqq\|h\|_{\infty} .
\end{gathered}
$$

It follows that $f$ is in $L_{1}\left(R^{n}\right)$. Hence, by (13), $f$ is in $\mathfrak{S}_{0}$ and $\left\{f_{r(\beta)}\right\}$ converges to $f$ in $\sigma\left(\mathfrak{H}_{0}, \mathfrak{H}_{0}^{\prime}\right)$. Evidently $f$ is in $B$. This proves that $B$ is $\sigma\left(\mathfrak{H}_{0}, \mathfrak{H}_{0}^{\prime}\right)$ - compact. Q.E.D.

For each $m \in N$, let

$$
\mathfrak{H}_{m}=\left\{f \in \mathfrak{H}_{0}: \theta_{s} . D^{w}(f) \in \mathfrak{H}_{0} \text { for all }(s, w) \in \mathfrak{P}(m)\right\} .
$$

Lemma. If $m \in N$ and $f \in \mathfrak{Y}_{m}$, then $\hat{f}$ is in $\mathfrak{S}_{m}$. 
Proof. For any $F \in \mathcal{S}^{\prime}$ and $(s, w) \in \mathfrak{P}(m)$, we have the well known equality

$$
\left[\theta_{s} \cdot D^{w}(F)\right]^{\wedge}=(-1)^{|w|} D^{s}\left(\theta_{w} \cdot \hat{F}\right) \text {. }
$$

Since $f^{*}$ is in $\mathfrak{S}_{m}$, Hormander's rule for differentiating products yields that $D^{s}\left(\theta_{w} . f^{*}\right)$ is in $\mathfrak{H}_{0}$ for each $(s, w) \in \mathfrak{P}(m)$. It follows from (16) that

$$
\left[\theta_{s} \cdot D^{w}(\hat{f})\right]^{\wedge}=(-1)^{|w|} D^{s}\left(\theta_{w} \cdot f^{*}\right)
$$

is in $\mathfrak{S}_{0}$ for all $(s, w) \in \mathfrak{P}(m)$. Hence, $\hat{f}$ is in $\mathfrak{S}_{m}$. Q.E.D.

For each $m \in N$, define the norm \|\|$(m)$ on $\mathfrak{H}_{m}$ by

$$
\|f\|^{(m)}=\frac{1}{2} \sum_{(s, w) \in \mathfrak{P}(m)}\left(\left\|\theta_{s} \cdot D^{w}(f)\right\|^{(0)}+\left\|\theta_{s} \cdot D^{w}(\hat{f})\right\|^{(0)}\right) .
$$

Note that, for each $m \in N$ and $f \in \mathfrak{H}_{m+1}$,

$$
\mathfrak{S}_{0} \supset \mathfrak{S}_{m} \supset \mathfrak{H}_{m+1} \text { and }\|f\|^{(0)} \leqq\|f\|^{(m)} \leqq\|f\|^{(m+1)} .
$$

Theorem 4. For each $m \in N$, the Fourier Transform is a surjective linear isometry on $\mathfrak{H}_{m}$.

Proof. That the Fourier Transform is isometric follows from (9) and (17). That it is surjective follows from (8) and the fact that $\mathfrak{H}_{m} \subset \mathfrak{H}_{0}$. Q.E.D.

Theorem 5. For each $m \in N, \mathfrak{Y}_{m} \subset C^{m}\left(R^{n}\right)$.

Proof. Since $\mathfrak{H}_{m} \subset \mathfrak{H}_{0} \subset L_{1}\left(R^{n}\right)$, and since each $f \in \mathfrak{H}_{m}$ is the Fourier transform of $\hat{f}^{*}$, we may differentiate under the integral sign, Q.E.D.

Theorem 6. For each $m \in N, \mathfrak{H}_{m}$ is a Banach space.

Proof. Let $\left\{f_{n}\right\}$ be any Cauchy sequence in $\mathfrak{S}_{m}$. Since $\mathfrak{Y}_{0}$ is complete, for each $(s, w) \in \mathfrak{P}(m)$ there exist functions $f_{s, w}$ and $h_{s, w}$ in $\mathfrak{S}_{0}$ for which

$$
\lim _{n}\left(\left\|f_{s, w}-\theta_{s} \cdot D^{w}\left(f_{n}\right)\right\|^{(0)}+\left\|h_{s, w}-\theta_{s} \cdot D^{w}\left(\hat{f}_{n}\right)\right\|^{(0)}\right)=0 ;
$$

by (18) and (14), this implies

$$
\lim _{n}\left\|f_{s, w}-\theta_{s} \cdot D^{w}\left(f_{n}\right)\right\|_{\infty}=0=\lim _{n}\left\|h_{s, w}-\theta_{s} \cdot D^{w}\left(\hat{f}_{n}\right)\right\|_{\infty} .
$$

It follows that, for all $(s, w) \in \mathfrak{P}(m), f_{s, w}=\theta_{s} . D^{w}\left(f_{0,0}\right)$ and $h_{s, w}=\theta_{s} \cdot D^{w}\left(h_{0,0}\right)$. Since

$$
\lim _{n}\left\|f_{n}-f_{0,0}\right\|^{(0)}=0=\lim _{n}\left\|\hat{f}_{n}-h_{0,0}\right\|^{(0)}
$$


it follows that $\hat{f}_{0,0}=h_{0,0}$. Hence,

$$
\lim _{n}\left\|f_{n}-f_{0,0}\right\|(m)=0 \text {. Q.E.D. }
$$

Theorem 7. For each $m \in N, \mathfrak{H}_{m}$ is reflexive.

Proof. Let $\mathfrak{O}(m)$ be a copy of $\mathfrak{P}(m)$ and define $\Psi \mid \mathfrak{H}_{m} \rightarrow \mathfrak{H}_{0}^{\mathfrak{P}(m) \cup \mathfrak{D}(m)}$ by letting, for each $f \in \mathfrak{H}_{m}, \Psi_{f}$ be the function on $\mathfrak{P}(m) \cup \mathfrak{D}(m)$ such that $\Psi_{f}(s, w)=\theta_{s} . D^{w}(f)$ for all $(s, w) \in \mathfrak{P}(m)$ and $\Psi_{f}(s, w)=\theta_{s} . D^{w}(\hat{f})$ for all $(s, w) \in \mathfrak{D}(m)$. Then $\Psi$ is a topological isomorphism of $\mathfrak{H}_{m}$ into $\mathfrak{S}_{0}^{\mathfrak{B ( m}) \cup \mathfrak{D}(m)}$. Since $\mathfrak{H}_{m}$ is a Banach space, $\Psi\left(\mathfrak{H}_{m}\right)$ is closed. Since products of reflexive spaces are reflexive ([9] p. 146), Theorem 3 implies that $\mathfrak{H}_{0}^{\mathfrak{B}(m) \cup \mathfrak{D}(m)}$ is reflexive. Since closed subspaces of semi-reflexive spaces are semi-reflexive, $\Psi\left(\mathfrak{H}_{m}\right)$ and $\mathfrak{H}_{m}$ are semi-reflexive. Since $\mathfrak{H}_{m}$ is a Banach space, it is reflexive. Q.E.D.

Theorem 8. Let $m$ be in $N$ and let $\mathfrak{D}(m)$ be a copy of $\mathfrak{P}(m)$. For each function $\varrho \mid \mathfrak{P}(m) \cup \mathfrak{D}(m) \rightarrow L_{\infty}\left(R^{n}\right)$, let $F_{\varrho} \mid \mathfrak{H}_{m} \rightarrow C$ be defined by

$$
F_{\varrho}(f)=\sum_{\substack{(s, w) \in \mathfrak{B}(m) \\(r, t) \in \mathfrak{D}(m)}} \int_{R^{n}}\left[\varrho(r, t) \cdot \theta_{r} D^{t}(\hat{f})+\varrho(s, w) \cdot \theta_{s} D^{w}(f)\right] \mathrm{d} \lambda .
$$

Then $\mathfrak{S}_{m}^{\prime}=\left\{F_{\varrho}: \varrho \mid \mathfrak{P}(m) \cup \mathfrak{D}(m) \rightarrow L_{\infty}\left(R^{n}\right)\right\}$.

Proof. Recall the mapping $\Psi$ defined in the proof of Theorem 6. It follows from Theorem 2 and the properties of $\Psi$ that Theorem 7 holds. Q.E.D.

Lemma. Let $w$ be any element of $\mathfrak{M}$. For each $x \in R^{n}$, write $m(x)$ for the number $\max \left\{\left|x_{j}\right|: j=1,2, \ldots, n\right\}$. The number $|w|$ is, by definition, $\sum_{j=1}^{n} w_{j}$. For all $x$ and $y$ in $R^{n}$ for which $m(x) \leqq 1$,

$$
\left|\theta_{w}(y+x)-\theta_{w}(y)\right| \leqq m(x) \cdot(2 \pi)^{|w|} \cdot|w|^{n} \cdot 2^{|w|} \cdot\left(m(y)^{|w|}+1\right) .
$$

Proof. Let $\Gamma$ be the set of all choice functions $\Psi$ in the product $\prod_{j=1}^{n}\left\{0,1, \ldots, w_{j}\right\}$ such that $m(\Psi) \neq 0$. Then

$$
\begin{gathered}
\left|\theta_{w}(y+x)-\theta_{w}(y)\right|=(2 \pi)^{|w|} \prod_{j=1}^{n} \sum_{k=0}^{w_{j}}\left(\begin{array}{c}
w_{j} \\
k
\end{array}\right) y_{j}^{w_{j}-k} x_{j}^{k}-\prod_{j=1}^{n} y_{j}^{w_{j}} \leqq \\
(2 \pi)^{|w|} \cdot \sum_{\Psi \in \Gamma} \prod_{j=1}^{n}\left(\begin{array}{c}
w_{j} \\
\Psi_{j}
\end{array}\right)\left|y_{j}\right|^{w_{j}-\Psi_{j}}\left|x_{j}\right|^{\Psi_{j}} \leqq(2 \pi)^{|w|} \sum_{\Psi_{\in} \Gamma} \prod_{j=1}^{n} w_{j}^{n} \cdot\left(m(y)^{w_{j}}+1\right) \cdot m(x) \leqq \\
\leqq(2 \pi)^{|w|} \cdot\left(m(y)^{|w|}+1\right) \cdot m(x) \cdot|w|^{\mid n} 2^{|w|} \text { Q.E.D. }
\end{gathered}
$$


Lemma. Let $w$ be in $\mathfrak{M}$ and $f$ in $\mathfrak{H}_{|w|}$. Then

$$
\int_{R^{n}}\left|f(y) \cdot m(y)^{|w|}\right| \mathrm{d} y<\infty
$$

(where $m(y)=\max \left\{\left|y_{j}\right|: j=1, \ldots, m\right\}$ ).

Proof. For each $j=1,2, \ldots, n$, let $w^{(j)}$ be the element of $\mathfrak{M}$ such that $w_{k}^{(j)}=|w|$ if $j=k$ and $w_{k}^{(j)}=0$ otherwise. Then, for each $y \in R^{n}$,

$$
\sum_{j=1}^{n}\left|\theta_{w(j)}(y)\right|=\sum_{j=1}^{n}\left|y_{j}\right|^{|w|} \cdot 2 \pi>m(y)^{|w|} .
$$

Thus, (5) and (15) imply (21). Q.E.D.

Lemma. Let $\left\{g_{k}\right\}$ be a non-negative sequence in $L_{1}\left(R^{n}\right)$ such that $\int g_{k} \mathrm{~d} \lambda=1$ for all $k \in N$ and $g_{k}(x)=0$ for all $x \in R^{n}$ farther from 0 than $1 / k$. Let $s$ be in $\mathfrak{M}$ and $f$ in $\mathfrak{H}_{|s|}$. Then

$$
\lim _{k}\left\|\left(g_{k} * f\right) \cdot \theta_{s}-f \cdot \theta_{s}\right\|_{1}=0 .
$$

Proof. For each $r>0$, let $B(r)$ be the ball of radius $r$ in $R^{n}$. Let $\varepsilon$ be any positive number. By (21), we may select $r>0$ such that

$$
\int_{R^{n} \backslash B(r)}\left|\theta_{s} \cdot f\right| \mathrm{d} \lambda<\varepsilon / 4
$$

and so, for $x \in B(1)$,

(ii) $\quad\left(\frac{1}{\sqrt{ }(2 \pi)}\right)^{n} \int_{B(r+1)^{c}}\left|\theta_{s}(y-x) \cdot f(y-x)\right| \mathrm{d} y \leqq \int_{B(r)^{c}}\left|\theta_{s} \cdot f\right| \mathrm{d} \lambda<\varepsilon / 4$.

Evidently, since $f$ is in $L_{1}\left(R^{n}\right)$,

$$
\lim _{x \rightarrow 0} \int_{R^{n}}|f(y-x)-f(y)| \mathrm{d} y=0
$$

so there exists $m \in N$ such that, if $x \in B(1 / m)$,

$$
\left(\frac{1}{\sqrt{ }(2 \pi)}\right)^{n} \int_{B(r+1)}\left|\theta_{s}(y)\right| \cdot|f(y-x)-| f(y) \mid \mathrm{d} y<\varepsilon / 4 .
$$

In view of (20) and (21), $m$ can be chosen so that

$$
\begin{aligned}
& \left(\frac{1}{\sqrt{ }(2 \pi)}\right)^{n} \int_{B(r+1)^{c}}\left|\theta_{s}(y)-\theta_{s}(y-x)\right||f(y-x)| \mathrm{d} y \leqq \\
& \leqq\left(\frac{1}{\sqrt{ }(2 \pi)}\right)^{n} \int_{R^{n}}\left|\theta_{s}(y+x)-\theta_{s}(y)\right| \cdot|f(y)| \mathrm{d} y<\varepsilon / 4
\end{aligned}
$$


whenever $x \in B(1 / m)$. From (i), (ii), (iii), and (iv) we have

(v)

$$
\begin{aligned}
& \left(\frac{1}{\sqrt{ }(2 \pi)}\right)^{n} \int_{R^{n}}\left|\theta_{s}(y)\right| \cdot|f(y-x)-f(y)| \mathrm{d} y \leqq \\
\leqq & \int_{B(r+1)}+\int_{B(r+1)^{c}}<\varepsilon / 4+\varepsilon / 4+\varepsilon / 4+\varepsilon / 4=\varepsilon .
\end{aligned}
$$

For all $k>m,(\mathrm{v})$ yields

$$
\begin{gathered}
\left\|\left(g_{k} * f\right) \cdot \theta_{s}-f \cdot \theta_{s}\right\|_{1}=\left(\frac{1}{2 \pi}\right)^{n} \int_{R^{n}}\left|\theta_{s}(y) \int_{R^{n}} g_{k}(x) \cdot f(y-x) \mathrm{d} x-f(y) \cdot \theta_{s}(y)\right| \mathrm{d} y= \\
=\left(\frac{1}{2 \pi}\right)^{n} \int_{R^{n}}\left|\theta_{s}(y) \int_{k}(x)[f(y-x)-f(y)] \mathrm{d} x\right| \mathrm{d} y \leqq \\
\leqq\left(\frac{1}{2 \pi}\right)^{n} \int_{B(1 / m)}\left|g_{k}(x)\right| \int_{R}\left|\theta_{s}(y)\right| \cdot|f(y-x)-f(y)| \mathrm{d} y \mathrm{~d} x< \\
<\left(\frac{1}{\sqrt{(2 \pi}}\right)^{n} \int_{B(1 / m)} g_{k}(x) \cdot \varepsilon \mathrm{d} x=\varepsilon .
\end{gathered}
$$

Q.E.D.

Theorem 9. For each $m \in Z^{+}, \mathfrak{H}_{m}$ is an algebra and $\mathfrak{S}$ a dense subset of $\mathfrak{H}_{m}$.

Proof. Let $f$ and $h$ be in $\mathfrak{S}_{m}$ and $(s, w)$ in $\mathfrak{P}(m)$. By Hörmander's Rule

$$
\theta_{s} D^{w}(f h)=\sum_{r \leqq w} \theta_{s} \cdot i^{-|w|}\left(\begin{array}{l}
w \\
r
\end{array}\right) \cdot D^{r}(f) \cdot D^{w-r}(h) ;
$$

since $(s, r)$ and $(0, w-r)$ are in $\mathfrak{P}(m)$, and since $\mathfrak{Y}_{0}$ is an algebra, $\theta_{s} D^{w}(f g)$ is in $\mathfrak{Y}_{0}$. Hence, $f$ is in $\mathfrak{S}_{m}$ and $\mathfrak{S}_{m}$ is an algebra.

For each $k=1,2, \ldots$ define

Thus

$$
g_{k} \mid R^{n} \ni x \rightarrow k^{1 / 2 n} \exp \left(-k \pi|x|^{2}\right) .
$$

$$
g_{k}=\hat{g}_{k} \mid R^{n} \ni x \rightarrow \exp \left(-\frac{\pi}{k}|x|^{2}\right)
$$

If $n=1$ and $p$ and $q$ are positive integers, then $\theta_{p} D^{q} \hat{g}_{k}$ is a linear combination $\sum_{j=0}^{q} l_{k, j}$ where

$$
l_{k, j}(x)=W_{j}\left(\frac{1}{k}\right) x^{j+p} \exp \left(-\frac{\pi}{k} x^{2}\right)
$$


and each $W_{j}$ is a polynomial of degree not exceeding $j$. The solution to $\mathrm{d} l_{k, j}(x) / \mathrm{d} x=0$ being $\sqrt{ }[k(j+p)] / 2 \pi$, we have

$$
\left\|l_{k, j}\right\|_{\infty}=W_{j}\left(\frac{1}{k}\right) \frac{k(j+p)^{(j+p) / 2}}{2 \pi} \cdot \exp \left(-\frac{k(j+p)}{2}\right) .
$$

This proves the first part of the following equality; the second part following from the first in view of the arbitrariness of $p$ :

$$
\lim _{k \rightarrow \infty}\left\|\theta_{p} D^{q} \hat{g}_{k}\right\|_{\infty}=0=\lim _{k \rightarrow \infty}\left\|\theta_{p} D^{q} \hat{g}_{k}\right\|_{1} .
$$

Evidently (ii) holds as well for arbitrary $n$, whenever $p$ and $q$ are multi-indices and $q \neq 0$. A direct calculation shows that, for $q \neq 0$,

$$
\lim _{k \rightarrow \infty}\left\|\theta_{q} g_{k}\right\|_{1}=0 \text {. }
$$

That

$$
\lim _{k \rightarrow \infty}\left\|g_{k} * f-f\right\|_{1}=0
$$

for all $f \in L_{1}\left(R^{n}\right)$ is well-known.

Let $h \in \mathfrak{S}_{m}$ and $(s, w) \in \mathfrak{P}_{m}$ be arbitrary. Then (ii) yields

$$
\begin{gathered}
\varlimsup_{k \rightarrow \infty}\left\|\theta_{s} D^{w}\left(\hat{g}_{k} h-h\right)\right\|_{1} \leqq \\
\leqq \varlimsup_{k \rightarrow \infty}\left(\sum_{0 \neq r \leqq w}\left(\begin{array}{l}
w \\
r
\end{array}\right)\left\|\theta_{s} D^{r}\left(\hat{g}_{k}\right) \cdot D^{w-r}(h)\right\|_{1}+\left\|\theta_{s} \cdot\left(\hat{g}_{k}-1\right) \cdot D^{w}(h)\right\|_{1}\right) \leqq \\
\leqq \varlimsup_{k \rightarrow \infty}\left(\sum_{0 \neq r \leqq w}\right)\left(\begin{array}{l}
w \\
r
\end{array}\right)\left\|D^{w-r}(h)\right\|_{1} \cdot\left\|\theta_{s} D^{r}\left(\hat{g}_{k}\right)\right\|_{\infty}+\left\|\theta_{s} D^{w}(h)\right\|_{1}\left\|\hat{g}_{k}-1\right\|_{\infty}=0 ;
\end{gathered}
$$

furthermore (iii) and (iv) imply

$$
\begin{gathered}
\varlimsup_{k \rightarrow \infty}\left\|\left(\theta_{s} D^{w}\left(\hat{g}_{k} h-h\right)\right)^{\wedge}\right\|_{1}= \\
=\varlimsup_{k \rightarrow \infty}\left\|\left(\theta_{s}\left[\left(\sum_{r \leqq w} i^{-|w|}\left(\begin{array}{c}
w \\
r
\end{array}\right) D^{r}\left(\hat{g}_{k}\right) \cdot D^{w-r} h\right)-D^{w} h\right]\right)^{\wedge}\right\|_{1}= \\
=\varlimsup_{k \rightarrow \infty}\left\|\sum_{r \leqq w} i^{-|w|} D^{s}\left(\left[\left(\begin{array}{l}
w \\
r
\end{array}\right)\left(\theta_{r} \cdot g_{k}\right) *\left(\theta_{w-r} \hat{h}\right)\right]-\theta_{w} \hat{h}\right)\right\|_{1} \leqq \\
\leqq \varlimsup_{k \rightarrow \infty} \sum_{0 \neq r \leqq w}\left(\begin{array}{c}
w \\
r
\end{array}\right)\left\|\theta_{r} g_{k} * D^{s}\left(\theta_{w-r} \hat{h}\right)\right\|_{1}+\left\|g_{k} * D^{s}\left(\theta_{w} \hat{h}\right)-D\left(\theta_{w} \hat{h}\right)\right\|_{1} \leqq \\
\leqq \varlimsup_{k \rightarrow \infty} \sum_{0 \neq r \leqq w}\left(\begin{array}{c}
w \\
r
\end{array}\right)\left\|\theta_{r} g_{k}\right\|_{1}\left\|D^{s}\left(\theta_{w-r} \hat{h}\right)\right\|_{1}+0=0 .
\end{gathered}
$$


This means that

$$
\lim _{k \rightarrow \infty}\left\|\hat{g}_{k} h-h\right\|^{(m)}=0 .
$$

Since $h \in \mathfrak{H}_{m}$ was arbitrary, we may apply the Fourier transformation to (v) to obtain

$$
\lim _{k \rightarrow \infty}\left\|g_{k} * h-h\right\|^{(m)}=0 .
$$

For each $k=1,2, \ldots$, we use (vi) to choose $k^{\prime}$ such that

$$
\left\|g_{k^{\prime}} *\left(\hat{g}_{k} h\right)-\hat{g}_{k} h\right\|^{(m)}<\frac{1}{m} .
$$

From this and (v) follows

$$
\lim _{k}\left\|g_{k^{\prime}} *\left(\hat{g}_{k} \cdot h\right)-h\right\|^{(m)}=0 .
$$

For any poor $(p, q)$ of multi-indices, we have

$$
\begin{gathered}
\left.\left\|\left(\theta_{p} D^{q}\left(g_{k^{\prime}} *\left(\hat{g}_{k} \cdot h\right)\right)\right)^{\wedge}\right\|_{1}=\| D^{p}\left(\theta_{q} \cdot \hat{g}_{k^{\prime}} \cdot\left(g_{k} * \hat{h}\right)\right)\right) \|_{1} \leqq \\
\leqq \sum_{r \leqq p}\left(\begin{array}{c}
p \\
r
\end{array}\right)\left\|D^{r}\left(\theta_{q} \hat{g}_{k^{\prime}}\right) \cdot\left[D^{p-r}\left(g_{k}\right) * \hat{h}\right]\right\|_{1} \leqq \\
\leqq \sum_{r \leqq p}\left(\begin{array}{l}
p \\
r
\end{array}\right)\left\|D^{r}\left(\theta_{q} \hat{g}_{k^{\prime}}\right)\right\|_{\infty}\left\|D^{p-r}\left(g_{k}\right)\right\|_{1}\|\hat{h}\|_{1}<\infty,
\end{gathered}
$$

which implies that $g_{k} *\left(\hat{g}_{k}, h\right) \in \mathfrak{S}$ for each $k=1,2, \ldots$.

Thus, (vii) implies that $\mathfrak{S}$ is dense in $\mathfrak{S}_{m}$. Q.E.D.

Theorem 10. The projective limit $\bigcap_{m=0}^{\infty} \mathfrak{H}_{m}$ is $\Im$.

Proof. By Theorem 9, $\mathfrak{S} \subset \bigcap_{m=0}^{\infty} \mathfrak{Y}_{m}$. It is a simple consequence of (15) and (14) that any function in $\bigcap_{m=0}^{\infty} \mathfrak{H}_{m}$ is rapidly decreasing. It is evident that the usual topology on $\mathfrak{S}$, that generated by the semi-norms of $(0)$, is the one generated by the norms \|\|$^{(m)}, m \in Z^{+}$. Q.E.D.

We have now shown that (1), (2), and (3) hold for the sequence $\left\{\mathfrak{H}_{m}\right\}_{m=0}^{\infty}$. Note that the duals of a sequence $\left\{H_{m}\right\}_{m=0}^{\infty}$ satisfying (1), (2), and (3) are Banach spaces under their strong topologies. Kucera noted in [4] that the sequence $\left\{H_{m}^{\prime}\right\}_{m=0}^{\infty}$ of duals may be regarded as an increasing chain such that $\mathcal{S}^{\prime}=\bigcup_{m=0}^{\infty} H_{m}^{\prime}$ and that the inductive topology induced on $\mathfrak{S}^{\prime}$ is finer than the strong topology $\beta\left(\mathbb{S}^{\prime}, \mathfrak{S}\right)$. Howev- 
er, the Mackey topology $\tau\left(\mathfrak{\Im}^{\prime}, \mathfrak{\Im}\right)$ on $\mathfrak{\Xi}^{\prime}$ is just $\beta\left(\mathfrak{\Xi}^{\prime}, \mathfrak{\Im}\right)$ and it is shown in [9] IV.4.4 that $\tau\left(\Im^{\prime}, \mathfrak{S}\right)$ is the inductive topology induced by the Mackey topologies $\tau\left(H_{m}^{\prime}, H_{m}\right)$. The Banach spaces $H_{m}^{\prime}$ bear their Mackey topologies precisely when they are reflexive, and this is the case when the spaces $H_{m}$ are reflexive. We have shown

Theorem 11. If a sequence $\left\{H_{m}\right\}_{m=0}^{\infty}$ satisfying (1), (2), and (3) is composed of reflexive spaces, then the strong topology $\beta\left(\Im^{\prime}, \mathbb{\Xi}\right)$ on $\mathfrak{\Xi}^{\prime}$ is the natural inductive topology on $\bigcup_{m=0}^{\infty} H_{m}^{\prime}$.

We will investigate the multiplication operators from $\mathfrak{S}_{p}$ and $\mathfrak{S}_{q}$ in a further paper.

\section{Bibliography}

[1] Hewitt, E. and Ross, K.: Abstract Harmonic Analysis, Vol. 1. Berlin: Springer Verlag. 1963.

[2] Hewitt, E. and Ross, K.: Abstract Harmonic Analysis, Vol. 2. Berlin: Springer Verlag. 1970.

[3] Kucera, J.: Fourier $L_{2}$-transform of distributions. Czech. Math. J. 19, 143-153 (1969).

[4] Kucera, J.: On multipliers of temperate distributions. Czech. Math. J. 21, 610-618 (1971).

[5] Kucera, J. and McKennon, K.: Certain topologies on the space of temperate distributions and its multipliers. Indiana Math. J. 24, 773-775 (1975).

[6] Kucera, J. and McKennon, K.: The topology on certain spaces of multipliers. Rocky Mountain J. of Math. 7, 377-383 (1977).

[7] Larsen, R.: An Introduction To The Theory Of Multipliers. Berlin: Springer Verlag. 1971.

[8] McKennon, K.: Multipliers, positive functionals, positive-definite functions, and FourierStieltjes transforms. Memoirs A.M.S., III, 1971.

[9] Schaeffer, H.: Topological Vector Spaces. New York: MacMillan Company 1966.

[10] Yosida, K.: Functional Analysis. Berlin: Springer Verlag 1968.

Author's address: Washington State University, Department of Mathematics, Pullman, Washington 99163, U.S.A. 